\title{
Diversidad arbórea en cercas vivas y dos fragmentos de bosque en la comunidad de Santa Adelaida, Estelí
}

\section{Pablo Siles*, Jorge Martinez Rayo **, Flavia Andino Rugama*** y Leonel Molina ${ }^{* * * *}$}

\section{Recibido: junio de 2013 / Aceptado: agosto de 2013}

Las cercas vivas son sistemas agroforestales utilizados por productores y promovidos por instituciones y organismos de desarrollo. Éstas cumplen con diferentes funciones de conservación, especialmente en paisajes fragmentados. En este estudio se presenta la diversidad arbórea de cercas vivas comparándola con dos fragmentos de bosques intervenidos en el trópico seco de Estelí, Nicaragua. Las familias más especiosas en el presente estudio fueron Mimosaceae, Moraceae, Fabaceae, Meliaceae, Bignonaceae y Boraginaceae, siendo, Leguminosae (Mimosaceae, Fabaceae y Caesalpinaceae) el grupo con más especies e individuos con respecto a las otras familias encontradas. Las especies más abundantes en el estudio fueron Bursera simaruba, Guazuma ulmifolia, Trichilia havanensis, Lysiloma divaricatum y Diospyros salicifolia. Las cercas vivas estudiadas presentan una alta diversidad florística. Sin embargo, muestran un alto porcentaje de especies introducidas y especies reproducidas asexualmente. Consideramos que las cercas vivas presentes en esta localidad tienen un rol potencial en la conservación de especies arbóreas (y posiblemente en la fauna), pero presentan una contribución menor en la provisión de servicios y productos

\footnotetext{
* Bioversity International Turrialba, Costa Rica. CATIE 7071. Correo electrónico: p.siles@cgiar.org

** Instituto Nicaragüense de Tecnología Agropecuaria (INTA), Universidad Nacional de Ingeniería (UNI) y Universidad Católica Agropecuaria del Trópico Seco (UCATSE), Estelí, Nicaragua. Correo electrónico: jmartinazaret@yahoo.com,jmartin@catie.ac.cr

*** Universidad Católica Agropecuaria del Trópico Seco (UCATSE), Esteli, Nicaragua. Correo electrónico: flavandino@gmail.com; flavia@ucatse.edu.ni 
a los productores. Por ende, ofrecen la oportunidad de incrementar y mejorar el aporte hacia productores por medio de cambios en la composición florística.

Palabras clave: bosque seco tropical / fragmento de bosque / sistemas agro forestales / especies arbóreas

\section{Introducción}

El bosque seco tropical (BST) es uno de los ecosistemas que caracterizan la zona del Pacífico de Nicaragua. Este ecosistema se caracteriza por temperaturas altas y relativamente estables con promedios de entre 21 y $30^{\circ} \mathrm{C}$, precipitaciones anuales de entre 800 y $1500 \mathrm{~mm}$ por año, y estaciones secas prolongadas de cinco a ocho meses. Esta combinación de calor y precipitación produce una evaporación potencial mayor que la precipitación. Contando con suficiente calor y una fertilidad del suelo más o menos aceptable, la falta de agua define la dinámica de los bosques del trópico seco (Murphy \& Lugo, 1995).

Este ecosistema (BST) está altamente amenazado por la actividad humana, resultando en una alteración del paisaje provocada por la agricultura, que en Centroamérica data de al menos 5,000 años (Gillespie, Grijalva \& Farris, 2000; Murphy \& Lugo, 1995). En Nicaragua, especialmente, la población está asentada en las zonas de vida correspondientes a bosques secos tropicales, lo que acentúa más la vulnerabilidad de estos bosques. Según las estadísticas de la FAO, la superficie boscosa total de Nicaragua representa el 39\% del territorio nacional (FAO, 2005). Sin embargo, parte de este porcentaje corresponde a bosque natural con grados variables de modificación causada por actividad humana, haciendo que la capacidad de estos bosques para generar servicios eco-sistémicos se reduzca.

Los sistemas agroforestales como los silvopastoriles, sistemas agroforestales con cultivos perennes (café, musáceas, cacao), huertos caseros y cercas vivas, pueden albergar una cantidad de especies arbóreas que compense los efectos negativos de la fragmentación y degradación de los bosque secos (Otero \& Onaindia, 2009). Todas las tecnologías agroforestales cuentan con un gran potencial para la conservación de la biodiversidad local y regional, particularmente de las especies vegetales nativas. Debido a su estructura y permanencia, las cercas vivas, en numerosas ocasiones, constituyen una de las piezas claves en las estrategias de conservación de la biodiversidad nativa y regional, en medio de sistemas de producción homogéneos (Pulido-Santacruz \& Renjifo, 2011).

Ante el problema del cambio climático y la pérdida de diversidad biológica producto de la deforestación, es de suma importancia realizar estudios sobre el impacto de tecnologías agroforestales, especialmente la de cercas vivas, en la preservación de la biodiversidad. Por tal razón, el presente estudio describe la diversidad de especies leñosas existentes en cercas vivas comparadas con fragmentos de bosques intervenidos, generando información sobre las especies más utilizadas en estos sistemas. Así, el estudio trata de identificar oportunidades de conservación y restauración en paisajes fragmentados, permitiendo comparar el potencial de conservación de las cercas vivas con el de los fragmentos de bosque. 


\section{Materiales y métodos}

El estudio se llevó a cabo en dos fragmentos de bosque seco y ocho cercas vivas ubicadas en la zona Pacífico Central de Nicaragua, en el departamento de Estelí $\left(13^{\circ} 03^{\prime} \mathrm{N}, 86^{\circ} 17^{\prime} \mathrm{W}\right)$. Esta zona presenta una temperatura media anual de $21.5^{\circ} \mathrm{C}$ y recibe de 800 a $900 \mathrm{~mm}$ de precipitación anual. El tipo de zona de vida está definido como bosque seco tropical según la clasificación de zonas de vida de Holdridge (1996).

El primer fragmento (F1) tiene un área de 2.9 ha y se ubica en la finca Los Chilamates, propiedad de la Universidad Católica Agropecuaria del Trópico Seco de Estelí (UCATSE). Éste presenta suelos arcillosos y de una fertilidad intermedia. El segundo fragmento (F2) tiene un área de 1.8 ha y se ubica en la finca Santa Adelaida, también propiedad de la UCATSE. Presenta un suelo arcilloso, pedregoso y de menor fertilidad, con pendiente de aproximadamente 35\%. Los dos fragmentos de bosque son pequeños y aislados en una matriz de pastizales y cultivos, y han sido intervenidos por personas que buscan leña para subsistencia. Por otro lado, las ocho cercas vivas consideradas para este estudio pertenecen a la comunidad de Santa Adelaida y fueron seleccionadas en base a tres criterios: la disponibilidad del productor para llevar a cabo el estudio, la presencia de cercas vivas de al menos 500 m de longitud y el acceso fácil.

En cada fragmento de bosque se establecieron tres parcelas con un tamaño de $1000 \mathrm{~m}^{2}(20$ × $50 \mathrm{~m})$. Cada parcela se dividió en 10 subparcelas de $100 \mathrm{~m}^{2}(20$ x 5 m) y se registró el nombre de la especie y su dap (diámetro a la altura del pecho) para todos los individuos con dap > a $2.5 \mathrm{~cm}$ y $3 \mathrm{~m}$ de altura, siguiendo la metodología de Sánchez-Merlo y colaboradores (Sánchez-Merlo et al., 2005a, 2005b). La unidad de muestreo en las cercas vivas consistió en transeptos de $500 \mathrm{~m}$ lineales por $2 \mathrm{~m}$ de ancho para un tamaño de $1000 \mathrm{~m}^{2}(2 \times 500 \mathrm{~m})$. Cada transepto fue dividido en 10 sub-transeptos de $50 \mathrm{~m}$ ( 2 x $50 \mathrm{~m})$. Se registró el nombre de la especie y su dap para todos los individuos con dap > a $2.5 \mathrm{~cm}$ y $3 \mathrm{~m}$ de altura, cuyo tallo estuviera dentro de un metro de la línea central del transepto.

La identificación de las especies arbóreas se realizó en el campo y con la ayuda de guías dendrológicas (Acevedo-Rodríguez, 2005; Gentry, 1993a; Holdridge \& Poveda, 1997; Jardim, Killeen \& Fuentes, 2003; Keller, 2003; Killen, García \& Beck, 1998; Ulloa \& Jorgensen, 1993; Zamora, 2000; Zamora, Jiménez \& Poveda, 2000, 2003). Las especies que no pudieron ser identificadas en campo fueron colectadas con material fértil para su identificación posterior con ayuda de las guías dendrológicas mencionadas anteriormente o fueron llevadas al herbario nacional ubicado en la Universidad Centroamericana (UCA) para ser identificadas con la ayuda del botánico Alfredo Grijalva.

Curvas de acumulación de especies, así como estimadores no-paramétricos de diversidad fueron realizados mediante el programa EstimateS v.5 (Colwell, 1997). Para cada parcela o transepto lineal el Índice de Valor de Importancia (IVI) fue computado en base a los valores relativos del área basal, densidad y frecuencia de las especies (Louman, Valerio \& Jiménez, 2001) en las subparcelas y sub-transeptos. Un análisis de conglomerados fue realizado con los valores de IVI en SAS release 9.1 
(SAS, 2004) utilizando el procedimiento PROC CLUSTER método Ward distancia Euclidiana. Un análisis de varianza (ANOVA) fue realizado para determinar diferencias entre los fragmentos de bosque $(\mathrm{F} 1, \mathrm{~F} 2)$ y las cercas vivas para las variables de densidad de individuos, área basal y especies presentes por parcela (especies en $1000 \mathrm{~m}^{2}$ ). Adicionalmente, un análisis de regresión lineal entre las especies por parcela y la densidad de individuos o el area basal fue llevado a cabo utilizando el procedimiento PROC REG en SAS release 9.1.

\section{Resultados}

En el estudio se contabilizaron 43 familias leñosas, representadas en 85 géneros y 106 especies, con un total de 1807 individuos con dap mayor a $2.5 \mathrm{~cm}$. Las familias con mayor número de especies fueron Mimosaceae (13 especies, 9 géneros), Moraceae (9 especies, 3 géneros), Fabaceae (6 especies, 6 géneros), Meliaceae (6 especies, 4 géneros), Bignonaceae ( 5 especies, 5 géneros) y Boraginaceae (4 especies, 2 géneros). Las especies más abundantes fueron Bursera simaruba (10.5\% del total de individuos), Guazuma ulmifolia (8.4\%), Trichilia havanensis (6.7\%), Lysiloma divaricatum (6.4\%) y Diospyros salicifolia (6.7\%). Estas cinco especies arbóreas representan en total $38 \%$ de todos los individuos encontrados en los dos tipos de vegetación (cercas vivas y fragmentos de bosque), mientras de todas las 106 especies encontradas, solamente 15 especies presentaron más de 30 individuos con el $68 \%$ de los individuos (ver Cuadro 1).

Al menos 26 especies (25\% del total de especies) presentaron baja abundancia (1.4\% del total de individuos) con sólo un individuo, a pesar de ser especies comúnmente encontradas en diversos sistemas del trópico seco, tales como Prestonia mexicana, Byttneria aculeata, Calycophyllum candidissimum, Canavalia aff villosa, Chloroleucon mangense, Citrus $\times$ aurantium, Cordia dentata, Delonix regia, Diphysa americana, Inga vera, Hibiscus sp, Malvaviscus sp, Jacaranda mimosifolia, Melicoccus bijugatus, Plumeria rubra, Prosopis juliflora, Sapindus saponaria, Sapium glandulosum, Varronia aff macrocephla, Trichilia martiana, Vitis tilifolia, Serjania aff grosii, Ficus aff trigonata, Ficus aff cotinifolia, Ficus sp y Senegalia aff picachensis. 
Cuadro 1. Resumen de las 15 especies más comunes en cercas vivas y dos fragmentos de bosque en la comunidad de Santa Adelaida, Estelí, Nicaragua (en base a 1807 individuos con dap $>2.5 \mathrm{~cm}$ )

\begin{tabular}{|c|c|c|c|c|c|}
\hline \multirow[b]{2}{*}{ Especie } & \multicolumn{3}{|c|}{ Tipo de vegetación } & \multirow[b]{2}{*}{ Total } & \multirow[b]{2}{*}{$\%$} \\
\hline & $\begin{array}{l}\text { Cercas } \\
\text { Vivas }\end{array}$ & $\begin{array}{l}\text { Fragmento } \\
1\end{array}$ & $\begin{array}{l}\text { Fragmento } \\
2\end{array}$ & & \\
\hline Bursera simaruba & 72 & 1 & 117 & 190 & 10.5 \\
\hline Guazuma ulmifolia & 73 & 31 & 47 & 151 & 8.4 \\
\hline Trichilia havanensis & & 121 & & 121 & 6.7 \\
\hline Lysiloma divaricatum & 8 & & 107 & 115 & 6.4 \\
\hline Diospyros salicifolia & 17 & 1 & 86 & 104 & 5.8 \\
\hline Karwinskia calderonii & 46 & & 48 & 94 & 5.2 \\
\hline Vachellia pennatula & 79 & & 5 & 84 & 4.6 \\
\hline Senna atomaria & 74 & & 10 & 84 & 4.6 \\
\hline Cascabela ovata & 27 & & 41 & 68 & 3.8 \\
\hline Cordia alliodora & 5 & & 47 & 52 & 2.9 \\
\hline Gliricidia sepium & 38 & & & 38 & 2.1 \\
\hline Opuntia cochenillifera & 34 & & & 34 & 1.9 \\
\hline Erythrina fusca & 2 & 31 & & 33 & 1.8 \\
\hline Jatropha curcas & & & 31 & 31 & 1.7 \\
\hline Pisonia macranthocarpa & 5 & 15 & 11 & 31 & 1.7 \\
\hline
\end{tabular}

El 57\% de todos los individuos muestreados presentaron diámetros de 2.5 a $8 \mathrm{~cm}$, y solamente el $4 \%$ de los individuos mostraron diámetros mayores a $40 \mathrm{~cm}$. El dap promedio de todos los individuos fue de $11.5 \pm 0.4 \mathrm{~cm}$. En ambos fragmentos de bosque (F1, F2) mostraron una distribución diamétrica de una "J" invertida muy marcada, indicando una mayor abundancia de individuos con diámetros menores. En cambio, las cercas vivas presentaron también una distribución de "J" invertida pero con mayor uniformidad en las categorías menores a $20 \mathrm{~cm}$, si se comparan con los fragmentos de bosque. Especialmente el F2 presentó un alto porcentaje de individuos con dap pequeños, donde el $77 \%$ de todos los individuos presentaron valores menores a $8 \mathrm{~cm}$ y con menos del $1 \%$ de los individuos con dap mayores a 40 $\mathrm{cm}$ (Ilustración 1).

Por el contrario, el F1 presentó mayor frecuencia de árboles mayores de 40 $\mathrm{cm}(11 \%)$ comparado con las cercas vivas $(4.5 \%)$ y el F2 (0.2\%). Adicionalmente, el F1 presenta hasta un $7 \%$ de sus individuos mayores de $72 \mathrm{~cm}$, siendo mayor al porcentaje acumulado de los otros hábitats. Este factor de distribución de diámetros es el factor principal por el cual en el F1 se presentan altos valores de área basal. 


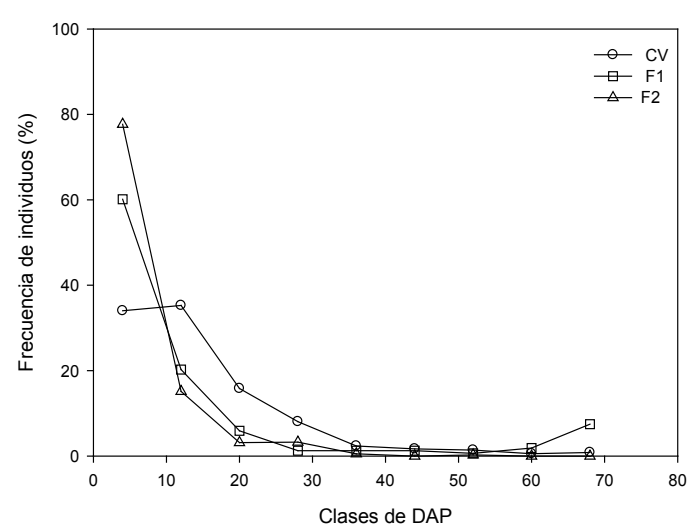

Ilustración 1. Frecuencia de clases diamétricas en cercas vivas y dos fragmentos de bosque en la comunidad de Santa Adelaida, Estelí, Nicaragua

Curvas de rarefacción basadas en individuos no mostraron diferencias en la riqueza de especies entre los fragmentos de bosque (ambos fragmentos combinados) y las cercas vivas (Ilustración 2a). Sin embargo, cuando se compara las curvas de rarefacción basadas en área muestreada (Ilustración 2b), se muestra efectivamente que los fragmentos de bosque presentan mayor riqueza de especies que las cercas vivas.

Adicionalmente, se observa que existe sólo una ligera diferencia entre la curva general de especies y la curva de los fragmentos de bosque, y ambas curvas están muy por encima de la curva de cercas vivas. En efecto, en la misma área efectiva de muestreo de 0.6 ha, el total general (todas las parcelas) presenta 84 especies, mientras los fragmentos de bosque 76 especies y las cercas vivas 60 especies. Se debe notar que el número total de especies encontradas en la curva general (todas las parcelas) basada tanto en individuos como en área de muestreo, presentó un incremento mayor a las curvas independientes de fragmentos de bosque o cercas vivas, lo que podría indicar una complementariedad en la diversidad total.

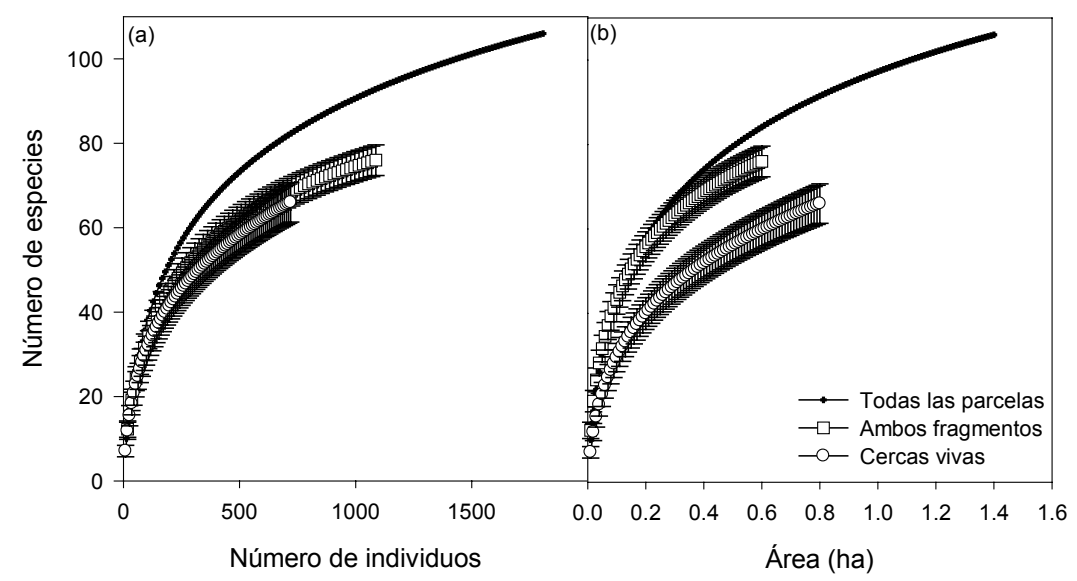

Ilustración 2. Curvas de rarefacción basadas en número de individuos y áreas para cercas vivas y dos fragmentos de bosque en la comunidad de Santa Adelaida, Estelí, Nicaragua 
La densidad de especies leñosas, así como el área basal y la riqueza de especies (géneros y familias) por parcela, presentó diferencias significativas entre las cercas vivas y cada uno de los fragmentos de bosque, lo que significa también que existen diferencias marcadas en términos de estructura (Cuadro 2). La riqueza promedio por parcela para las cercas vivas y el FI es de 21 (con 20 a 18 géneros y 15 familias) especies por $1000 \mathrm{~m}^{2}$, mientras que el F2 presentó valores de 36 especies (34 géneros y 25 familias). El F2 tiene mayor densidad de individuos comparado con el F1 y las cercas vivas ( $\mathrm{p}>0.001$ ), con valores promedios de 255 (F2), 107 (F1) y 90 (cercas vivas) por cada $1000 \mathrm{~m}^{2}$ respectivamente.

Sin embargo, a pesar de que el F1 presenta baja densidad, éste muestra un alto valor de área basal $\left(62 \mathrm{~m}^{2} \mathrm{ha}^{-1}\right)$ comparado con las cercas vivas $\left(25 \mathrm{~m}^{2} \mathrm{ha}^{-1}\right) \mathrm{o}$ el F2 $\left(17 \mathrm{~m}^{2} \mathrm{ha}^{-1}\right)$. La riqueza de especies en todas las unidades de muestreo varió extremadamente entre parcelas con 13 especies (cercas vivas) a parcelas ricas con 38 especies (F2). Esta variabilidad en riqueza de especies está altamente asociada a la densidad de individuos. De esta forma, las parcelas más ricas en especies presentan también la mayor densidad de individuos, y existe una relación lineal marcada $\left(r^{2}=0.86 p=0.0001\right.$, riqueza de especies vs densidad de individuos). Sin embargo, las parcelas con mayor área basal no presentaron mayor riqueza de especies, siendo esta relación no significativa $\left(\mathrm{r}^{2}=0.03\right)$.

Cuadro 2. Riqueza en cercas vivas y dos fragmentos de bosque en la comunidad de Santa Adelaida, Estelí, Nicaragua (en base a 1807 individuos con dap $>2.5 \mathrm{~cm}$ )

\begin{tabular}{|c|c|c|c|c|c|c|c|}
\hline & & \multicolumn{6}{|c|}{ Tipo de vegetación } \\
\hline & & \multicolumn{2}{|c|}{$\begin{array}{l}\text { Cercas } \\
\text { vivas }\end{array}$} & \multicolumn{2}{|c|}{ Fragmento 1} & \multicolumn{2}{|c|}{ Fragmento 2} \\
\hline \multicolumn{2}{|c|}{ No. especies totales } & \multicolumn{2}{|l|}{65} & \multicolumn{2}{|l|}{33} & \multicolumn{2}{|l|}{54} \\
\hline \multicolumn{2}{|c|}{ No. individuos totales } & \multicolumn{2}{|l|}{720} & \multicolumn{2}{|l|}{321} & \multicolumn{2}{|l|}{766} \\
\hline Individuos & $\left(\right.$ por $\left.1000 \mathrm{~m}^{2}\right)$ & 90 & (12) & 107 & (9) & 255 & (33) \\
\hline Área basal & $\left(\mathrm{m}^{2} \mathrm{ha}^{-1}\right)$ & 25.3 & (3.9) & 62.3 & (7.0) & 17.3 & (1.2) \\
\hline Especies & $\left(\right.$ por $\left.1000 \mathrm{~m}^{2}\right)$ & 21 & $(2.1)$ & 21 & $(0.7)$ & 36 & (1.5) \\
\hline Géneros & $\left(\right.$ por $\left.1000 \mathrm{~m}^{2}\right)$ & 20 & $(2.3)$ & 18 & $(0.6)$ & 34 & (1.8) \\
\hline Familias & $\left(\right.$ por $\left.1000 \mathrm{~m}^{2}\right)$ & 15 & (1.3) & 15 & $(0.9)$ & 25 & $(0.6)$ \\
\hline Alpha & $\left(\right.$ por $\left.1000 \mathrm{~m}^{2}\right)$ & 8.8 & $(0.8)$ & 8.0 & $(0.3)$ & 11.5 & $(0.4)$ \\
\hline Shannon & $\left(\right.$ por $\left.1000 \mathrm{~m}^{2}\right)$ & 2.5 & $(0.1)$ & 2.3 & $(0.1)$ & 2.8 & $(0.1)$ \\
\hline Simpson & $\left(\right.$ por $\left.1000 \mathrm{~m}^{2}\right)$ & 9.2 & $(0.8)$ & 5.7 & $(0.5)$ & 10.4 & (1.0) \\
\hline
\end{tabular}

Nota: Los valores entre paréntesis representan errores estándar.

Respecto a la similitud de especies entre las cercas vivas y los dos fragmentos de bosque (F1 y F2), se encontraron bajos valores de especies compartidas entre el F1 y los otros ecosistemas. La mayor cantidad de especies compartidas se encontró entre 
las cercas vivas y el F2 (34 especies), mientras que entre el F1 y las cercas vivas fue de 10 especies, y de 11 especies entre el F1 con respecto al F2. El índice cualitativo de Sørensen mostró que las cercas vivas y el F2 son los más parecidos en términos de la presencia de especies (0.57), mientras que el F1 mostró bajos valores de este índice con cercas vivas (0.20) y F2 (0.25). De la misma forma, los índices de MorisitaHorn y Bray-Curtis mostraron un patrón de similitud muy parecido al encontrado con el índice Sørensen, con mayor similitud entre las cercas vivas y el F2, pero baja similitud de ambos con el F1.

De las 106 especies encontradas en el estudio, solamente 10 especies ocurrieron en los tres tipos de hábitats (cercas vivas, F1, F2): Bursera simaruba, Guazuma ulmifolia, Diospyros salicifolia, Pisonia macranthocarpa, Tabebuia rosea, Eugenia acapulcensis, Myrospermum frutescens, Ruprechtia costata y Ceiba pentandra, las cuales representan el $9 \%$ del total de especies registradas.

Adicionalmente, muchas especies ocurrieron solamente en hábitats específicos. En el caso de F1, fueron 19 especies, lo que corresponde al 58\% de las 33 especies encontradas, entre las que están Trichilia havanensis, Trophis racemosa, Maclura tinctoria, Cinnamomum triplinerve, Genipa americana, Simarouba amara, Urera baccifera, Piper tuberculatum; Annona muricata, Inga vera, Sapium glandulosum, Trichilia martiana, Vitis tiliifolia, Canavalia aff villosa, Ficus aff trigonata, Ficus insipida, Ficus maxina, Ficus sp1 y Ficus sp2. Algunas de estas especies pertenecen a los géneros Ficus, Trophis, Cinnamomum, Genipa, Simarouba, Sapium e Inga, que son de importancia para la conservación (especies clave, keystone species, como en el caso de Ficus) por su producción de frutos para la fauna silvestre.

En lo que respecta al F2, 17 especies, es decir 31\% del total de las especies encontradas en ese hábitat, fueron específicas. Mayormente son éstas especies de crecimiento secundario, como Ipomoea praecana, Serjania triquetra, Serjania aff grosii, Pithecoctenium crucigerum, Jatropa curcas, Cochlospermun vitifolium, Lasianthaea fruticosa, Casearia sp, Cisus sp, Schoepfia schreberi, Prestonia mexicana, Byttneria aculeata, Calycophyllum candidissimum, Chloroleucon mangense, Hibiscus sp, Malvaviscus sp y Prosopis juliflora.

Por su parte, de las especies presentes solamente en las cercas vivas (específicas de las cercas vivas) hay dos grupos: especies nativas que son relativamente comunes en áreas de bosque secundario o primario, y especies exóticas o introducidas. En el primer grupo, la ausencia de estas especies en el F1 y F2 puede deberse a un artificio de muestreo. Estas especies son Gliricidia sepium, Acacia farnesiana, Enterolobium cyclocarpum, Leucaena leucocephala, Ficus aff cotinifolia, Ficus goldmanii, Diphysa americana, Senegalia aff picachensis, Alvaradoa amorphoides, Crescentia alata, Croton sp, Solanun sp, Cordia dentata, Varronia macrocephala, Melicoccus bijugatus, Sapindus saponaria y Plumeria rubra. Las especies introducidas corresponden al 19\% de las especies encontradas en las cercas vivas. Algunas de estas especies incluso presentaron una alta frecuencia, como es el caso de Opuntia cochenillifera y Synadenium grantii. Además de estas especies ya mencionadas, se citan especies introducidas específicas en las cercas vivas, como Eucalyptus camaldulensis, Yucca guatemalensis, Senna siamea, Azadirachta indica, Bougainvillea $x$ buttiana, Cupressus lusitanica, Citrus $\times$ aurantium, Delonix regia y Jacaranda mimosifolia. 
El análisis de conglomerados basado en los IVI de las especies, agrupó las parcelas muestreadas (parcelas + transeptos) en tres grupos diferentes florísticamente. Se utilizó el IVI de las especies y no solamente su frecuencia o presencia, ya que este valor indica, además de su composición florística (presencia y frecuencia de individuos), la dominancia de las especies presentes (área basal, densidad, frecuencia en las sub-parcelas).

El F1 presentó en sí mismo un sólo grupo diferente florísticamente a F2 y las cercas vivas, mientras un segundo grupo fue representado por las parcelas del F2 más un transepto de la cerca viva (CV4), y el tercer grupo fue conformado con los transeptos restantes de las cercas vivas (Ilustración 3). En términos de composición florística, el F1 se diferencia mucho de las parcelas de F2 y cercas vivas, pero existe una similitud muy fuerte entre las tres parcelas del F1. Las parcelas del F2 muestran diferencias de las cercas vivas pero estas diferencias son menos acentuadas si se comparan con las parcelas del F1.

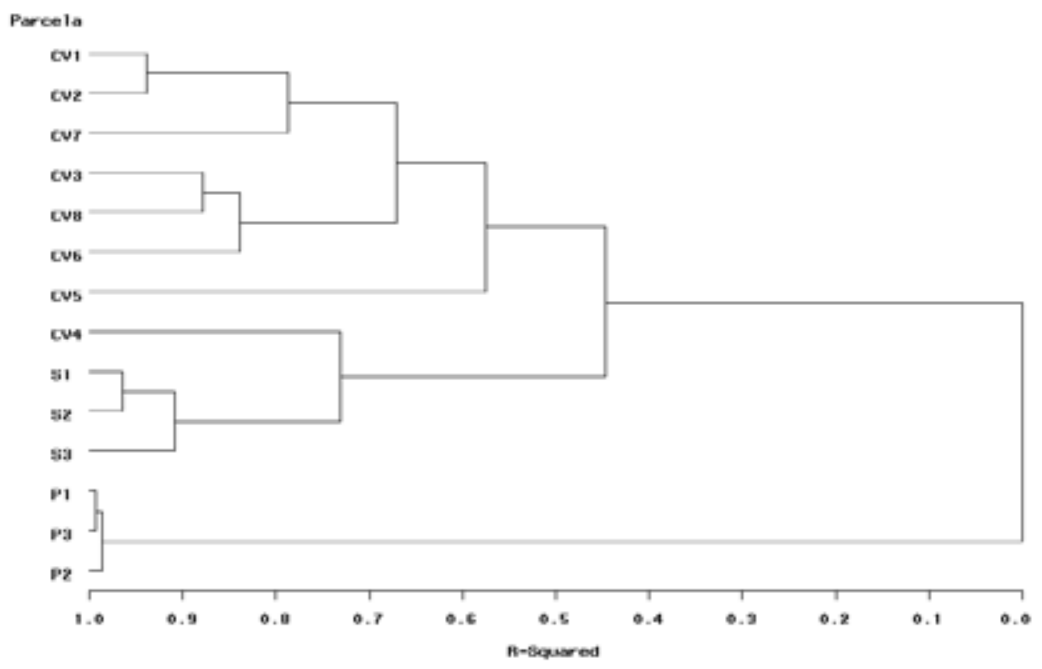

Ilustración 3. Dendrograma de clasificación de las unidades de vegetación muestreadas y agrupadas por similitud florística. Análisis de conglomerado basado en el IVI (Índice de valor de importancia) de cada especie (P denota el Fragmento 1, $\mathrm{S}$ denota el Fragmento 2 y CV denota cercas vivas)

Ahora bien, las diez especies más importantes (IVI más altos) difieren entre los tipos de hábitat, siendo esto lo que define las agrupaciones por composición florística (Cuadro 3). Solamente G. ulmifolia está presente con alto IVI en los tres tipos de hábitats, siendo una de las tres especies dominantes de la vegetación. En el F1 Erythrina fusca es la especie dominante (33\%), con algunas especies ausentes de los otros hábitats, que en total acumulan un IVI de 248. La dominancia de estas especies, y no sólo su presencia, es la razón por la cual este fragmento de bosque difiere significativamente de las cercas vivas y del F2.

En el F2 B. simaruba, G. ulmifolia, L. divaricatum, K. calderonii y D. salicifolia son las especies dominantes con mayor IVI (145.8), de las cuales dos especies son también 
dominantes en las cercas vivas (B. simaruba, G. ulmifolia). Sin embargo, Vachellia pennatula, Senna atomaria y Enterolobium cyclocarpum tienden a ser dominantes en las cercas vivas y reemplazan la dominancia de $L$. divaricatum, $K$. calderonii y $D$. salicifolia. Adicionalmente, S. purpurea y G. sepium son dominantes en las cercas vivas y coincidentemente ambas especies son reproducidas fácilmente por medios vegetativos.

Cuadro 3. Índice de Valor de Importancia (IVI) por composición florística en cercas vivas y dos fragmentos de bosque en la comunidad de Santa Adelaida, Estelí, Nicaragua

\begin{tabular}{llllll}
\hline Cercas vivas & & F1 & & F2 & \\
\hline Especie & IVI & Especie & IVI & Especie & IVI \\
\hline Guazuma ulmifolia & 41.3 & Erythrina fusca & 100.2 & Bursera simaruba & 40.9 \\
Vachellia pennatula & 29.4 & Trichilia havanensis & 56.9 & Guazuma ulmifolia & 32.2 \\
Senna atomaria & 27.7 & Guazuma ulmifolia & 24.3 & Lysiloma divaricatum & 27.8 \\
Bursera simaruba & 25.7 & Cordia sp & 15.1 & Karwinskia calderonii & 23.0 \\
Enterolobium & & & & & \\
cyclocarpum & 21.4 & Pisonia macranthocarpa & 11.3 & Diospyros salicifolia & 21.9 \\
Karwinskia calderonii & 18.8 & Maclura tinctoria & 10.8 & Cascabela ovata & 15.4 \\
Spondias purpurea & 9.7 & Ficus insípida & 10.1 & Cordia alliodora & 11.1 \\
Gliricidia sepium & 9.6 & Tabebuia rosea & 9.7 & Jatropha curcas & 10.2 \\
Platymiscium & & & & Co chlo sper m u n & \\
parviflorum & 8.3 & Trophis racemosa & 8.8 & vitifolium & 9.4 \\
Otras & 108.1 & Otras & 52.8 & Otras & 108.0 \\
\hline
\end{tabular}

\section{Discusión}

\subsection{Diversidad en los hábitats estudiados}

A nivel de diversidad de familias de los hábitats incluidos en este estudio se puede mencionar que los resultados son consistentes con otros estudios realizados en el trópico seco (Gentry, 1995; Gillespie et al., 2000). Las familias más especiosas fueron Mimosaceae, Moraceae, Fabaceae, Meliaceae, Bignonaceae y Boraginaceae. Sin embargo, sumando las familias Mimosaceae, Fabaceae y Caesalpinaceae como especies leguminosas en general, este grupo dobla en especies (23 especies) e individuos ( $27 \%$ de los individuos) a cualquier otra familia encontrada en este estudio. Gentry (1995) mostró que la Leguminosae (sensu lato Fabaceae, Mimosaceae y Caesalpinaceae) y la Bignonaceae son las familias más dominantes del bosque seco en el neotrópico. Respectivamente, la familia Leguminosae es dominante en especies arbóreas, mientras que la familia Bignonaceae en especies de lianas.

En lo que respecta a lianas, el estudio mostró presencia baja en términos de especies, individuos e IVI, y su mayor densidad fue en el F2. Por mucho, las tres 
especies de lianas más prevalecientes fueron Pithecoctenium crucigerum (Bignonaceae), Serjania triquetra (Sapindaceae) e Ipomoea praecana (Convulvulaceae). De hecho, la baja presencia de lianas en todas las parcelas puede ser la causa por la cual la familia Bignonaceae es la cuarta familia más especiosa si se considera a Fabaceae, Mimosaceae y Caesalpinaceae como un solo grupo Leguminosae.

Las otras familias más especiosas que la Bignonaceae (Moraceae y Meliaceae) presentaron mayor número de especies pero menor número de géneros, con el género Ficus en Moraceae y Trichilia en Meliaceae con alto número de especies. Gentry (1995) menciona también Rubiaceae, Sapindaceae, Euphorbiaceae, Flacourtiaceae y Capparidaceae como las cinco familias más predominantes en el trópico seco, seguidas de Leguminosae y Bignonaceae. Sin embargo, para Centro América, Gillespie et al. (2000) mencionan a Sapindaceae como la tercera familia más especiosa, seguida de Rubiaceae y Euphorbiaceae. En nuestro estudio Euphorbiaceae está presente como una familia especiosa pero solamente después de la familia Boraginaceae.

A nivel de géneros, Gentry (1995) menciona Tabebuia, Casearia, Trichilia, Erythroxilum, Arrabidaea, Randia, Capparis, Bursera, Vachellia (Acacia) y Coccoloba como los géneros más difundidos en el trópico seco. En el set de datos completo del estudio, al menos seis de estos géneros están presentes (Tabebuia, Casearia, Trichilia, Randia, Capparis, Bursera, Vachellia (Acacia)) y al menos Capparis fue encontrado ocasionalmente fuera de las parcelas y transeptos de muestreo. Adicionalmente, Gentry (1995) menciona géneros como Bursera, Caesalpinia y Lonchocarpus como sobre-representados en Centro América, de los cuales Bursera está representado por dos especies (B. simarouba y B. tomentosa) en nuestro estudio. Pero B. simarouba es la especie con más individuos y la menos presente en los tres hábitats muestreados. Esta especie fue también la única presente en siete bosques secos a lo largo de Centro América (Gillespie et al., 2000). Similarmente, en cercas vivas estudiadas en cuatro paisajes ganaderos (dos en Costa Rica y dos en Nicaragua), esta especie resultó ser la más abundante en las localidades de Cañas en Costa Rica y Matiguás en Nicaragua (Harvey et al., 2003). En general, B. simarouba es apreciada por su rápido crecimiento y adaptación, a pesar de su mala calidad para ser empleada como leña por considerarse muy porosa, lo que produce humo y cenizas negras. No obstante, sus rebrotes jóvenes son consumidos por el ganado y sus frutos son consumidos por las urracas (Calocitta formosa) y los chocoyos (Aratinga spp.), según conocimiento local de los ganaderos de la zona (Martínez-Rayo, 2003).

En cuanto a las lianas, en todas las parcelas muestreadas se encontraron solamente 12 especies pertenecientes a nueve familias. De éstas, tres especies fueron encontradas en cercas vivas (Gouania lupuloides, Rhamnaceae; Ipomoea aff clavata, Convolvulaceae; Senegalia aff picachensis, Mimosaceae), dos especies en el F1 (Vitis tiliifolia, Vitaceae; Canavalia aff villosa, Fabaceae) y nueve especies en el F2 (Serjania triquetra, Serjania aff grosii, Sapindaceae; Ipomoea aff clavata, Ipomoea praecana, Convolvulaceae; Pithecoctenium crucigerum, Bignonaceae; Cisus sp, Vitaceae; Prestonia mexicana, Apocynaceae; Byttneria aculeata, Sterculiaceae; Gouania lupuloides, Rhamnaceae). La baja presencia de lianas en todas las parcelas indica fragmentos de bosque muy alterados por la actividad humana, considerando que en muestras bosques secos $\left(1000 \mathrm{~m}^{2}\right)$ alcanzan entre 10 y 25 especies (Gentry, 1995). Las lianas son consideradas como indicadores fisionómicos de bosques maduros debido a que 
en general son más abundantes y diversas en bosques jóvenes, pero su área basal total se mantiene constante a diferentes edades, por lo que el área basal individual aumenta con la edad del bosque (lianas con diámetros mayores a $10 \mathrm{~cm}$ ) (Dewalt, Schnitzer \& Denslow, 2000).

\subsection{Comparación de densidad, área basal y especie en los hábitats estudiados}

Se encontraron diferencias marcadas en la estructura de la vegetación y la riqueza de especies entre los hábitats (cercas vivas, F1, F2). En términos de densidad de individuos con DAP mayores a $2.5 \mathrm{~cm}$, la mayor densidad se encontró en el F2, seguido del F1 y finalmente de las cercas vivas, mientras el área basal presentó mayores valores en F1, con valores inferiores para cercas vivas y F2. Estos resultados son ligeramente diferentes de los encontrados por Sánchez-Merlo et al. (2005b), donde las cercas vivas presentaron mayores densidades y áreas basales en comparación a los bosques secundarios, bosques riparios, charrales y potreros de baja y alta densidad.

En nuestro estudio las cercas vivas presentaron valores inferiores en densidad que los otros hábitats, pero valores intermedios de área basal. El F2 mostró altos valores de densidad y a veces especies con tallos múltiples (Diospyros salicifolia), pero una alta proporción de estos individuos con diámetros pequeños. En términos generales nuestros estimados de densidades se aproximan a los mostrados por Harvey et al. (2003), donde se muestran valores de entre 240 a 67 individuos por kilómetro lineal de cerca viva con diámetros mayores de $10 \mathrm{~cm}$ y valores de $414 \mathrm{a}$ 148 individuos, que incluyen árboles con diámetros menores a $10 \mathrm{~cm}$ (Harvey et al., 2003). Si consideramos nuestro valor estimado de densidad de 180 individuos por kilómetro lineal (90 individuos por transepto de $500 \mathrm{~m}$ lineales), sería un valor intermedio de los encontrados por Harvey et al. (2003) y similar a los encontrados en Matiguás, Nicaragua (35 árboles en 100 m de cerca viva).

Con respecto a los fragmentos de bosque, los valores de densidad para individuos con diámetros mayores a $2.5 \mathrm{~cm}$ están en los rangos de valor encontrados por Gillespie et al. (2000), quienes muestran valores de 135 a 264 individuos por $1000 \mathrm{~m}^{2}$ para bosques secos en América Central, y muy por debajo de los encontrados por Gentry (1995) para otros bosques secos del neo-trópico (especialmente para Suramérica y México). Sin embargo, el área basal del F1 muestra valores muy por encima de los encontrados en el trabajo de Gillespie et al. (2000), de 18 a $25 \mathrm{~m}^{2} \mathrm{ha}^{-1}$, los que son más similares a los encontrados en el F2.

Adicionalmente, se nota una diferencia marcada en la composición de especies entre los dos fragmentos (ver discusión adelante). Murphy y Lugo (1995) muestran valores de área basal para bosque secos de Centroamérica y el Caribe que varían entre 17 y más de $40 \mathrm{~m}^{2} \mathrm{ha}^{-1}$, mientras los valores de bosques húmedos alcanzan hasta $75 \mathrm{~m}^{-2} \mathrm{ha}^{-1}$. Por lo tanto, el F1 mostraría valores por encima de los bosques secos, lo que puede ser explicado por la presencia de suelos más profundos en esta área, así como una menor pendiente, que puede indicar mayor presencia de humedad durante los meses más secos, lo que reduciría el efecto de sequía.

El F2 presentó la mayor riqueza y diversidad de especies comparado con las cercas vivas y el F1. El promedio de la riqueza de especies en las parcelas del F2 fue de 36 , mientras que el F1 y las cercas vivas presentaron un promedio de 21 especies. De 
hecho, ambos fragmentos de bosque presentaron valores de riqueza de especies por debajo de los encontrados por Gillespie et al. (2000) usando el método de transeptos de Gentry (Gentry, 1993b), quien para Centroamérica muestra valores de entre 45 y 75 especies por $1000 \mathrm{~m}^{2}$. De hecho, estos dos fragmentos de bosque son sujetos a un alto grado de alteración antrópica, como quemas, extracción de madera y leña, e introducción de animales para pastoreo de forma accidental, lo que puede bajar significativamente la riqueza de especies.

Por otro lado, las cercas vivas presentaron valores de riqueza de especies por transepto comparables con los fragmentos de bosque, así como altos valores de especies totales encontradas (65 especies totales en las ocho cercas vivas). Estos resultados son relevantes desde un punto de vista de conservación pues muestran la baja presencia de cercas vivas mono-específicas, y sugieren que la presencia de especies encontradas podría aumentar con un mayor esfuerzo de muestreo. Sin embargo, se debe mencionar el alto porcentaje (19\%) de especies introducidas en estas cercas. De especial mención son Opuntia cochenillifera y Synadenium grantii, que fueron encontradas en densidades relativamente altas en algunas cercas. Adicionalmente, las cercas vivas muestran una alta frecuencia (y dominancia) de especies con reproducción asexual (O. cochenillifera, S. grantii, Y. guatemalensis, S. purpurea, G. sepium y B. simaruba), lo que claramente muestra que la composición botánica de estas cercas se debe a preferencias y decisiones de productores. El caso de Gliricidia sepium, que es una especie típica del trópico seco, llama la atención pues fue una especie abundante en las cercas vivas pero estuvo ausente en los dos fragmentos de bosque, lo que induce a pensar que su alta frecuencia en el paisaje se debe a factores antropogénicos.

La especie Guazuma ulmifolia es la especie más dominante en las cercas vivas estudiadas. Esta es una especie ampliamente utilizada en potreros y cercas vivas porque presenta diversos usos - tales como leña, carbón, madera y alimentación para el ganado -, presenta una buena regeneración y soporta condiciones de suelo amplias. La especie Bursera simarouba se caracteriza por ser uno de los árboles con gran frecuencia en el establecimiento de cercas vivas en Centroamérica, ya que presenta cierta tolerancia al estrés hídrico y fácil reproducción por medio de estacas. Esta especie es citada adicionalmente por poseer propiedades medicinales, pero representa una fuente de madera y leña de baja calidad. Estas características probablemente determinen su gran uso por parte de los productores para establecer cercas vivas.

El análisis de conglomerado mostró una similitud entre las cercas vivas y el F2. Esto puede indicar que las cercas vivas diversas presentan una alta frecuencia de árboles presentes también en bosques jóvenes y charrales, adicionales a las especies establecidas específicamente por los productores. Así, éstas pueden jugar un papel importante en la conservación de la biodiversidad porque amplían la oferta de recursos y nichos para un amplio grupo de especies de plantas y mamíferos. Sin embargo, gran parte de la diversidad de las cercas vivas podría depender de su conexión a los remanentes del bosque, ya que las cercas conectadas al bosque tienen mayor probabilidad de presentar una mayor diversidad de especies arbóreas (y similitud con los bosques). Muchas de las especies encontradas en las cercas en nuestro estudio son especies de árboles de regeneración rápida (rápido crecimiento 
y alta producción de semilla), posiblemente con dispersión proveniente de los remanentes de bosque que son seleccionados por los productores. Por ejemplo, Vachellia pennatula, Senna atomaria, Karwinskia calderonii, Platymiscium parviflorum y Enterolobium cyclocarpum son claramente especies de bosques secundarios y charrales en bosques secos, y su presencia y dominancia en las cercas vivas puede deberse a la presencia de remanentes de bosque. En general, es poco probable que los productores se interesen por la siembra de estas especies, pero cuando éstas están presentes en las cercas por dispersión de semillas provenientes de fragmentos de bosque, es muy probable que los productores las dejen en las cercas.

Las cercas vivas tienen un papel importante en la conservacion de especies, sobre todo en paisajes fragmentados. En general, para aves y mariposas se han realizado estudios comparativos de cercas vivas multiestratos y simples (DeClerck, Martínez, \& DeClerck, 2011; Lang, Gormley, Harvey \& Sinclair, 2003; Sáenz, Villatoro, Ibrahim, Fajardo \& Pérez, 2007; Tobar \& Ibrahim, 2010). Éstos muestran que las cercas multiestratos presentan una mayor riqueza y abundancia de especies de mariposas y aves que las cercas vivas simples, ya que pueden ayudar a mantener el $56 \%$ de las especies encontradas en los bosques secundarios y ribereños. Para el caso de las aves, las cercas vivas sirven como puentes o conexiones dentro de las matrices agropecuarias, como hábitat de borde para aquellas especies que son capaces de forrajear dentro de potreros (pasturas), y como fuente de recursos alimenticios para especies que dependen de arboles para su alimentación (DeClerck et al., 2011). De esta forma se evidencia que, dependiendo del manejo que realizan los productores, las cercas vivas pueden jugar un importante papel para la conservación de mariposas en estos sistemas (Tobar \& Ibrahim, 2010).

De la misma forma, cuando se ha comparado cercas vivas de árboles nativos plantados con cercas de árboles exóticos o espontáneos, estas últimas mantienen una alta diversidad de comunidades de plantas (árboles y arbustos, 77 especies), así como de aves, con 98 especies (Otero \& Onaindia, 2009). Además, parece existir una correlación marcada entre la diversidad de plantas, la estructura de la cerca y la conectividad, con la riqueza de especies de aves que éstas mantienen. Esto se debe a que las aves usan las cercas para una variedad de propósitos y éstas, en conjunto con fragmentos de bosques, mantienen un arreglo de especies que son una sub-muestra de las especies que existían en el paisaje original.

Las cercas vivas, además de presentar una contribución a la conservación de organismos, son una práctica de productores con fines estratégicos dentro de los sistemas agrícolas. Su función principal es delimitar los límites de la finca y dividir lotes dentro de ésta. Sin embargo, a menudo se citan otros servicios y productos de las cercas vivas a los productores, tales como estacas vivas para las nuevas cercas, forraje, madera, leña y frutos, pero la importancia relativa de dichos productos es generalmente baja (Harvey et al. 2003).

En el caso del presente estudio, se puede observar que las especies con mayor importancia relativa (G. ulmifolia, V. pennatula, S. atomaria, B. simaruba, E. cyclocarpum, K. calderonii, S. purpurea, G. sepium y P. parviflorum) tienen poco potencial para proveer productos de importancia a los productores, a excepción de leña. Guazuma ulmifolia es mencionada como una especie de importancia para otros sistemas ganaderos ubicados en Miraflor, Estelí y Matiguás-Matagalpa, donde se reporta importancia 
por poseer buena calidad de leña y ser una especie forrajera (Casasola, Ibrahim, Harvey \& Kleinn, 2001; Martínez-Rayo, 2003). Sin embargo, es posible que estas cercas, además de brindar una contribución a la conservación de biodiversidad en paisajes fragmentados, al mismo tiempo puedan proveer a los productores más productos para que éstos mejoren sus condiciones de vida.

Esto implicaría tratar de modificar la composición de las cercas vivas en dos direcciones. Por ejemplo, las cercas que delimitan áreas cercanas a las viviendas (huertos caseros, cultivos anuales) podrían ser enriquecidas con especies frutales de alto potencial nutritivo, adaptadas a la zona. Las cercas ubicadas en otro tipo de lotes lejanos a las viviendas (como potreros) pueden ser enriquecidas con especies maderables y de leña de buena calidad. Este cambio en la composición florística de las cercas vivas, por otro lado, puede potencialmente presentar un compromiso entre la conservación y la provisión de más productos a los propietarios. Sin embargo, una cuidadosa selección de especies y formas de establecimiento debe ser tomada en cuenta como una estrategia de intensificación agroecológica para mantener una diversidad de especies y hábitats mientras incrementamos producción, ingresos y una mejor dieta a nivel del hogar.

\section{Conclusiones}

Las familias más especiosas en el presente estudio fueron Mimosaceae, Moraceae, Fabaceae, Meliáceae, Bignonaceae y Boraginaceae. Por consiguiente, cuando se combinan las familias Mimosaceae, Fabaceae y Caesalpinaceae como especies leguminosas, este grupo dobla en especies e individuos a las otras familias encontradas.

Desde un punto de vista de conservación, las cercas vivas estudiadas presentan una alta diversidad florística similar a la encontrada en otros estudios. Sin embargo, éstas presentan un alto porcentaje de especies introducidas (Opuntia cochenillifera y Synadenium grantii), así como de especies reproducidas asexualmente (O. cochenillifera, S. grantii, Y. guatemalensis, S. purpurea, G. sepium y B. simaruba).

La presencia y abundancia de especies como Vachellia pennatula, Senna atomaria, Karwinskia calderonii, Platymiscium parviflorum y Enterolobium cyclocarpum en las cercas vivas puede deberse a la presencia de remanentes de bosque cercanos a las cercas, ya que claramente estas especies son dominantes en bosque secundarios y charrales en bosques secos.

Aunque las cercas vivas presentes en esta localidad tienen potencialmente un rol en la conservación de especies arbóreas (y posiblemente en la fauna), éstas representan una contribución menor en proveer servicios y productos a los productores. Así, ofrecen la oportunidad de incrementar y mejorar el aporte a los productores por medio de cambios en su composición florística. 


\section{Referencias bibliográficas}

Acevedo-Rodríguez, P. (2005). Vines and Climbing plants of Puerto Rico and the Virgin Islands. Contributions from the United States National Herbarium, 51, $1-483$.

Casasola, F., Ibrahim, M., Harvey, C. \& Kleinn, C. (2001). Caracterización y productividad de sistemas silvopastoriles tradicionales en Moropotente, Estelí, Nicaragua. Agroforestería en las Américas, 8(30), 17-20.

Colwell, R. (1997). EstimateS: statistical estimation of species richness and shared species from samples. Versión 5: Departament of Ecology and Evolutionary Biology, University of Connecticut, U.S.A. Accesible en internet: http://viceroy.eeb. uconn.edu/estimates.

DeClerck, F., Martínez, A. \& DeClerck, R. (2011). Aves en cercas vivas. Conservación de biodiversidad en paisajes agrícolas: un desafío para la producción ganadera. Agroforestería en las Américas, 48, 21-25.

Dewalt, S. J., Schnitzer, S. A. \& Denslow, J. S. (2000). Density and diversity of lianas along a chronosequence in a central Panamanian lowland forest. Journal of Tropical Ecology, 16(1), 1-19.

FAO. (2005). Global forest resources assessment 2005 - Progess towards sustainable management. FAO Forest. Resources Assessment Working Paper 83. Rome: FAO.

Gentry, A. (1993a). A field guide to the families and genera of woody plants of Northwest South America (Colombia, Ecuador, Peru) with supplementary notes on herbaceous taxa; Illustrations by Rodolfo Vásquez. Chicago and London: Conservation International and the University of Chicago Press.

Gentry, A. (1993b). Patterns of diversity and floristic composition in Neotropical Montane Forests. En S. Churchill, H. Balslev, E. Forero \& J. Luteyn (Eds.). Biodiversity and Conservation of Neotropical Montane Forest. Proceedings of the Neotropical Montane Forest Biodiversity and Conservation Symposium. The New York Botanical Garden, 21-26 June 1993 (pp. 103-126). New York: The New York Botanical Garden.

Gentry, A. (1995). Diversity and floristic composition of neotropical dry forest. In S. H. Bullock, H. A. Mooney \& E. Medina (Eds.). Seasonally dry tropical forests (pp. 146-194). Cambridge: Cambridge University Press.

Gillespie, T., Grijalva, A. \& Farris, C. (2000). Diversity, composition, and structure of tropical dry forests in Central America. Plant Ecology, 147, 37-47.

Harvey, C. A., Villanueva, C., Villacis, J., Chacon, M., Munoz, D., Lopez, M., et al. (2003). Contribución de las cercas vivas a la productividad e integridad ecológica de los paisajes agricolas en America Central. Agroforesteria en las Americas, 10(39-40), 30-39.

Holdridge, L. (1996). Ecología basada en zonas de vida. San José, Costa Rica: IICA.

Holdridge, L. \& Poveda, L. (1997). Árboles de Costa Rica. Volumen I: Palmas y otras monocotiledóneas arbóreas y árboles de hojas compuestas y lobuladas. San Jose, Costas Rica: Centro Científico Tropical.

Jardim, A., Killeen, T. \& Fuentes, A. (2003). Guía de los árboles y arbustos del bosque seco Chiricano, Bolivia. Santa Cruz de la Sierra: FAN-Bolivia. 
Keller, R. (2003). Identification of tropical woody plants in the absence of flowers. A field guide (2a ed.). Berlin: Basel. Birkhauser Verlag.

Killen, T., García, E. \& Beck, S. (1998). Guia de árboles de Bolivia. Santa Cruz, Bolivia: Herbario Nacional de Bolivia-Missouri Botanical Garden.

Lang, I., Gormley, L. H., Harvey, C. A. \& Sinclair, F. L. (2003). Composición de la comunidad de aves en cercas vivas de Río Frío, Costa Rica. Agroforestería en las Américas, 10(39-40), 86-92.

Louman, B., Valerio, J. \& Jiménez, W. (2001). Bases ecológicas. En B. Louman, D. Quiros \& M. Nilsson (Eds.). Silvicultura de bosques latifoliados húmedos con énfasis en América Central. Manual técnico/ CATIE; no 46 (pp. 21-75). Turrialba: CATIE.

Martínez-Rayo, J. (2003). Conocimiento local de productores ganaderos sobre cobertura arbórea en la parte baja de la cuenca del rio Bulbul en Matiguas, Nicaragua. Tesis de Maestría no publicada. Centro Agronómico Tropical de Investigación y Enseñanza (CATIE), Turrialba, Costa Rica.

Murphy, P. \& Lugo, A. (1995). Dry forest of Central America and the Caribbean. In S. H. Bullock, H. A. Mooney \& E. Medina (Eds.). Seasonally dry tropical forests (pp. 9-33). Cambridge: Cambridge University Press.

Otero, J. \& Onaindia, M. (2009). Landscape structure and live fences in Andes Colombian agrosystems: upper basin of the Cane-Iguaque River. Revista de biología tropical, 57(4), 1183-1192.

Pulido-Santacruz, P. \& Renjifo, L. M. (2011). Live fences as tools for biodiversity conservation: A study case with birds and plants. Agroforestry systems, 81(1), 15-30.

Sáenz, J. C., Villatoro, F., Ibrahim, M., Fajardo, D. \& Pérez, M. (2007). Relación entre las comunidades de aves y la vegetación en agropaisajes dominados por la ganadería en Costa Rica, Nicaragua y Colombia. Agroforestería en las Américas, 45, 37-48.

Sánchez-Merlo, D., Harvey, C. A., Grijalva, A., Medina, A., Vílchez, S. \& Hernandez, B. (2005a). Diversidad, composicion y estructura de la vegetación en un agropaisaje ganadero en Matiguás, Nicaragua. Revista de biologia tropical, 53(34), 387-414.

Sánchez-Merlo, D., Harvey, C., Grijalva, A., Medina, A., Vílchez, S. \& Hernández, B. (2005b). Diversidad, composición y estructura de la vegetación en un paisaje fragmentado de bosque seco en Rivas, Nicaragua. Recursos Naturales y Ambiente, 45, 91-104.

SAS. (2004). SAS OnlineDoc ${ }^{\circledR}$ 9.1.3. Cary, NC, USA: SAS Institute Inc.

Tobar, D. E. \& Ibrahim, M. (2010). ¿Las cercas vivas ayudan a la conservación de la diversidad de mariposas en paisajes agropecuarios? Revista de Biología Tropical, 58(1), 447-463.

Ulloa, C. \& Jorgensen, P. (1993). Árboles y arbustos de los Andes del Ecuador. AAU reports, 30, 1-264.

Zamora, N. (2000). Árboles de la mosquitia hondurena: descripción de 150 especies. Turrialba: CATIE.

Zamora, N., Jiménez, Q. \& Poveda, L. (2000). Árboles de Costa Rica. Volumen II. Heredia, Costa Rica: INBIO-CCT-CI.

Zamora, N., Jiménez, Q. \& Poveda, L. (2003). Árboles de Costa Rica. Volumen III. Heredia, Costa Rica: INBIO-CCT-CI. 